\title{
Existence Result for a Superlinear Fractional Navier Boundary Value Problems
}

\author{
Habib Mâagli, Abdelwaheb Dhifli and Abdulah Khamis Alzahrani
}

\begin{abstract}
In this paper, we study the following fractional Navier boundary value problem

$$
\left\{\begin{array}{l}
D^{\beta}\left(D^{\alpha} u\right)(x)=u(x) g(u(x)), \quad x \in(0,1), \\
\lim _{x \longrightarrow 0} x^{1-\beta} D^{\alpha} u(x)=-a, \quad u(1)=b,
\end{array}\right.
$$

where $\alpha, \beta \in(0,1]$ such that $\alpha+\beta>1, D^{\beta}$ and $D^{\alpha}$ stand for the standard Riemann-Liouville fractional derivatives and $a, b$ are nonnegative constants such that $a+b>0$. The function $g$ is a nonnegative continuous function in $[0, \infty)$ that is required to satisfy some suitable integrability condition. Using estimates on the Green's function and a perturbation argument, we prove the existence of a unique positive continuous solution, which behaves like the unique solution of the homogeneous problem.
\end{abstract}

Mathematics Subject Classification. 34A08, 34B15, 34B18, 34B27.

Keywords. Fractional Navier differential equations, positive solutions, Green's function, perturbation arguments.

\section{Introduction}

Recently, many papers on fractional differential equations have been studied extensively by many researches. The motivation for those works stems from the fact that fractional equations serve as an excellent tool to describe many phenomena in various fields of science and engineering such as viscoelasticity, electrochemistry, control theory, porous media, electromagnetism and other fields. Also, it provides an excellent tool to describe the hereditary properties of various materials and processes. Concerning the development of theory methods and applications of fractional calculus, we refer to $[5,8-12,14,16,23$, $24,26,28]$ and the references therein for discussions of various applications.

The theory of fractional differential equations with various boundary conditions has been developed very quickly and the investigation for the 
existence, uniqueness and asymptotic behavior of positive continuous solutions attracted a considerable attention of researches ( see $[1-4,6,7,13,15,17-$ $19,21,22,25,29,30]$ and the references therein ).

In [18], Mâagli et al. studied the following initial value problem:

$$
\left\{\begin{array}{l}
D^{\alpha} u(x)=-p(x) u^{\sigma}, \quad x \in(0,1), \\
\lim _{x \longrightarrow 0^{+}} x^{1-\alpha} u(x)=0
\end{array}\right.
$$

where $\alpha \in(0,1), \sigma<1$ and $p$ is a nonnegative continuous function in $(0,1)$ satisfying some appropriate conditions related to the Karamata class $\mathcal{K}$ ( see Definition 4 below ). By a potential theory approach associated with $D^{\alpha}$ and some technical tools relying to Karamata regular variation theory, the authors proved the existence, uniqueness and asymptotic behavior of a positive solution to problem (1.1) in the weighted space of continuous functions $C_{1-\alpha}([0,1])$.

Later, in [19], Mâagli and Dhifli studied the following sublinear fractional Navier boundary value problem:

$$
\begin{cases}D^{\beta}\left(D^{\alpha} u\right)(x)=-p(x) u^{\sigma}, & x \in(0,1), \\ \lim _{x \longrightarrow 0^{+}} x^{1-\beta} D^{\alpha} u(x)=0, & u(1)=0,\end{cases}
$$

where $\sigma \in(-1,1), \alpha, \beta \in(0,1]$ such that $\alpha+\beta>1$ and $p$ is a nonnegative continuous function in $(0,1)$. Under some appropriate conditions on the function $p$ and using the Schäder fixed point theorem, the authors proved the existence of a unique positive solution to problem (1.2). Further, based on the asymptotic behavior for the Green function and some technical tools relying on Karamata regular variation theory, the authors gave a global asymptotic behavior of such solutions to problem (1.2).

Inspired by the above-mentioned papers, we aim at studying in this paper the following superlinear fractional Navier boundary value problem:

$$
\left\{\begin{array}{l}
D^{\beta}\left(D^{\alpha} u\right)(x)=u(x) g(u(x)), \quad x \in(0,1), \\
\lim _{x \longrightarrow 0^{+}} x^{1-\beta} D^{\alpha} u(x)=-a, \quad u(1)=b,
\end{array}\right.
$$

where $\alpha, \beta \in(0,1]$ such that $\alpha+\beta>1$ and $a$ and $b$ are nonnegative constants such that $a+b>0$. The nonlinear term $g(t)$ is required to be a nonnegative continuous function on $[0, \infty)$ satisfying some appropriate conditions related to the class of functions $\mathcal{K}_{\alpha, \beta}$ defined as follows.

Definition 1. Let $\alpha, \beta \in(0,1]$. A Borel measurable function $q$ in $(0,1)$ belongs to the class $\mathcal{K}_{\alpha, \beta}$ if $q$ satisfies the following:

$$
\int_{0}^{1} r^{\alpha-1}(1-r)^{\alpha+\beta-1}|q(r)| \mathrm{d} r<\infty .
$$

We use the properties of this class to investigate an existence result for the fractional Navier boundary value problem (1.3). To state our main result in this paper, we need to introduce some convenient notations. Throughout this paper, we denote $\mathcal{B}((0,1))$ the set of Borel measurable functions in $(0,1)$ and $\mathcal{B}^{+}((0,1))$ the set of nonnegatives ones. We use $C_{r}([0,1])$ to denote the 
set of continuous functions $f$ on $(0,1]$ such that $x \rightarrow x^{r} f(x)$ is continuous on $[0,1]$.

For $q \in \mathcal{B}((0,1))$, we denote

$$
\kappa_{q}:=\sup _{x, t \in(0,1)} \int_{0}^{1} \frac{H(x, r) H(r, t)}{H(x, t)}|q(r)| \mathrm{d} r,
$$

where $H(x, t)$ is the Green function of the operator $u \rightarrow-D^{\beta}\left(D^{\alpha} u\right)$ in $(0,1)$, with boundary conditions $\lim _{x \longrightarrow 0^{+}} x^{1-\beta} D^{\alpha} u(x)=0$ and $u(1)=0$. We will prove that if $q \in \mathcal{K}_{\alpha, \beta}$, then $\kappa_{q}<\infty$.

We denote by $\omega$ the unique solution of the following homogenous problem:

$$
\left\{\begin{array}{l}
D^{\beta}\left(D^{\alpha} u\right)(x)=0, \quad x \in(0,1), \\
\lim _{x \longrightarrow 0^{+}} x^{1-\beta} D^{\alpha} u(x)=-a, \quad u(1)=b .
\end{array}\right.
$$

We can easily verify that for $x \in(0,1)$

$$
\omega(x):=a h_{1}(x)+b h_{2}(x),
$$

where

$$
h_{1}(x)=\frac{\Gamma(\beta)}{\Gamma(\alpha+\beta)} x^{\alpha-1}\left(1-x^{\beta}\right) \text { and } h_{2}(x)=x^{\alpha-1} .
$$

Finally, a combination of the following hypotheses on the term $g$ is required:

$\left(\mathbf{H}_{1}\right) g$ is a nonnegative continuous function in $[0, \infty)$.

$\left(\mathbf{H}_{2}\right)$ There exists a nonnegative function $q \in \mathcal{K}_{\alpha, \beta} \cap C((0,1))$ satisfying:

(i) $q(t) \leq t^{-\mu+1-\alpha} L(t)$ for $t$ near 0 with $\mu \leq 1$ and $L \in \mathcal{K}$ satisfying $\int_{0}^{\eta} t^{-\mu} L(t) \mathrm{d} t<\infty$

(ii) $\kappa_{q} \leq \frac{1}{2}$.

(iii) For each $x \in(0,1)$, the map $t \rightarrow t(q(x)-g(t \omega(x)))$ is nondecreasing on $[0,1]$.

$\left(\mathbf{H}_{3}\right)$ The function $t \rightarrow \operatorname{tg}(t)$ is nondecreasing on $[0, \infty)$.

As a typical example of the function satisfying $\left(H_{1}\right)-\left(H_{3}\right)$, we quote $g(t)=t^{\sigma}$, where $\sigma \geq 0$.

Our main result is the following.

Theorem 1. Assume $\left(H_{1}\right)-\left(H_{2}\right)$, then problem (1.3) has a positive solution $u$ in $C_{1-\alpha}([0,1])$ satisfying

$$
c_{0} \omega(x) \leq u(x) \leq \omega(x), \quad x \in(0,1),
$$

where $c_{0}$ is a constant in $(0,1)$.

Moreover, this solution is unique if hypothesis $\left(H_{3}\right)$ is also satisfied.

Observe that in Theorem 1 , we obtain a positive solution $u \in C_{1-\alpha}([0,1])$ to problem (1.3) whose behavior is not affected by the perturbed term. That is, it behaves like the solution $\omega$ of the homogeneous problem (1.5).

Our paper is organized as follows. In Sect. 2, we give some estimates on $H(x, t)$. In Sect. 3, for a given function $q \in \mathcal{K}_{\alpha, \beta}$ with $\kappa_{q} \leq \frac{1}{2}$, we construct the Green function $\mathcal{H}(x, t)$ of the perturbed operator $u \rightarrow-D^{\beta}\left(D^{\alpha} u\right)+q(x) u$ with boundary conditions $\lim _{x \longrightarrow 0^{+}} x^{1-\beta} D^{\alpha} u(x)=0$ and $u(1)=0$ and we 
derive some of its properties. Exploiting these results, we prove our main result in Sect. 4.

\section{Definitions and Preliminary Results}

\subsection{Fractional Calculus}

For the convenience of the reader, we recall in the following some basic definitions and elementary properties of fractional calculus (For more details, see $[9,23,26])$.

Definition 2. The Riemann-Liouville fractional integral of order $\alpha>0$ of a function $f:(0,1) \longrightarrow \mathbb{R}$ is given by

$$
I^{\alpha} f(x)=\frac{1}{\Gamma(\alpha)} \int_{0}^{x}(x-t)^{\alpha-1} f(t) \mathrm{d} t
$$

provided that the right-hand side is pointwise defined on $(0,1)$.

Definition 3. The Riemann-Liouville fractional derivative of order $\alpha>0$ of a function $f \in \mathcal{B}((0,1))$ is given by

$$
D^{\alpha} f(x)=\frac{1}{\Gamma(n-\alpha)}\left(\frac{\mathrm{d}}{\mathrm{d} x}\right)^{n} \int_{0}^{x}(x-t)^{n-\alpha-1} f(t) \mathrm{d} t=\left(\frac{\mathrm{d}}{\mathrm{d} x}\right)^{n} I^{n-\alpha} f(x),
$$

where $n=[\alpha]+1$ and $[\alpha]$ mean the integer part of the number $\alpha$, provided that the right-hand side is pointwise defined on $(0,1)$.

Lemma $1([9,26])$.

(i) Let $\alpha, \beta>0$ and $0<a<1$. Let $f \in L^{1}((0, a)) \cap C((0, a))$, then we have

$$
\begin{gathered}
D^{\alpha} I^{\alpha} f(x)=f(x) \quad \text { for } x \in[0, a], \\
I^{\beta} I^{\alpha} f(x)=I^{\alpha+\beta} f(x) \quad \text { for } x \in[0, a], \beta+\alpha \geq 1 .
\end{gathered}
$$

(ii) Let $\alpha>0$ and $0<a<1$. Let $f \in L^{1}((0, a))$, then

$$
D^{\alpha} f(x)=0 \quad \text { if and only if } \quad f(x)=\sum_{k=1}^{n} c_{k} x^{\alpha-k},
$$

where $n$ is the smallest integer greater than or equal to $\alpha$ and $\left(c_{1}, \ldots, c_{n}\right)$ $\in \mathbb{R}^{n}$.

(iii) Let $\alpha>0$ and $0<a<1$. Let $f$ such that $D^{\alpha} f \in L^{1}((0, a)) \cap C((0, a))$, then

$$
I^{\alpha} D^{\alpha} f(x)=f(x)+\sum_{k=1}^{n} c_{k} x^{\alpha-k}
$$

where $n$ is the smallest integer greater than or equal to $\alpha$ and $\left(c_{1}, \ldots, c_{n}\right)$ $\in \mathbb{R}^{n}$. 


\subsection{Karamata Class $\mathcal{K}$}

In this subsection, we introduce the Karamata class $\mathcal{K}$ and we recall some fundamental properties of functions belonging to this class.

Definition 4. The class $\mathcal{K}$ is the set of Karamata functions $L$ defined on $(0, \eta]$ by

$$
L(t):=c \exp \left(\int_{t}^{\eta} \frac{z(s)}{s} \mathrm{~d} s\right)
$$

for some $\eta>1$, where $c>1$ and $z \in C([0, \eta])$ such that $z(0)=0$.

Remark 1. It is clear that a function $L$ is in $\mathcal{K}$ if and only if $L$ is a positive function in $C^{1}((0, \eta])$ for some $\eta>1$, such that $\lim _{t \longrightarrow 0^{+}} \frac{t L^{\prime}(t)}{L(t)}=0$.

As a typical example of function belonging to the class $\mathcal{K}$, we quote

$$
L(t)=\prod_{j=1}^{m}\left(\log \left(\frac{w}{t}\right)\right)^{\xi_{j}},
$$

where $\xi_{j}$ are real numbers, $\log _{j} x=\log \circ \log \ldots \log x(j$ times $)$ and $w$ is a sufficiently large positive real number such that $L$ is defined and positive on $(0, \eta]$ for some $\eta>1$.

Lemma 2 ([20,27]). Let $\gamma \in \mathbb{R}$ and $L$ be a function in $\mathcal{K}$ defined on $(0, \eta]$. Then, we have that

(i) if $\gamma>-1$, then $\int_{0}^{\eta} s^{\gamma} L(s) \mathrm{d} s$ converges and $\int_{0}^{t} s^{\gamma} L(s) \mathrm{d} s \underset{t \rightarrow 0^{+}}{\sim} \frac{t^{1+\gamma} L(t)}{\gamma+1}$;

(ii) if $\gamma<-1$, then $\int_{0}^{\eta} s^{\gamma} L(s) \mathrm{d} s$ diverges and $\int_{t}^{\eta} s^{\gamma} L(s) \mathrm{d} s \underset{t \rightarrow 0^{+}}{\sim}-\frac{t^{1+\gamma} L(t)}{\gamma+1}$.

Lemma 3 [19]. Let $\alpha, \beta \in(0,1]$. Let $f \in C((0,1))$ such that the map $t \rightarrow$ $(1-t)^{\alpha+\beta-1} f(t)$ is integrable and $|f(t)| \leq t^{-\delta} L(t)$ for $t$ near 0 , with $\delta \leq 1$ and $L \in \mathcal{K}$ satisfying $\int_{0}^{\eta} t^{-\delta} L(t) \mathrm{d} t<\infty$. Then the function $x \rightarrow I^{\beta} f(x) \in$ $C((0,1)) \cap L^{1}((0,1))$ and $\lim _{x \rightarrow 0} x^{1-\beta} I^{\beta} f(x)=0$.

\subsection{Estimates on the Green's Function $\mathrm{H}(\mathrm{x}, \mathrm{t})$}

This subsection is devoted to give estimates on the Green function $H(x, t)$. From [19], the Green function of the operator $u \rightarrow-D^{\beta}\left(D^{\alpha} u\right)$ in $(0,1)$, with boundary conditions $\lim _{x \longrightarrow 0^{+}} x^{1-\beta} D^{\alpha} u(x)=0$ and $u(1)=0$, is given explicitly by

$$
H(x, t)=\frac{x^{\alpha-1}(1-t)^{\alpha+\beta-1}-\left((x-t)^{+}\right)^{\alpha+\beta-1}}{\Gamma(\alpha+\beta)},
$$

where $\Gamma$ is the Euler gamma function and for $r \in \mathbb{R}, r^{+}=\max (r, 0)$.

First, we recall in the following lemma due to [19] some estimates on the Green function $H(x, t)$ and properties of the associated potential kernel defined by

$$
V f(x)=\int_{0}^{1} H(x, t) f(t) \mathrm{d} t, \quad \text { for } f \in B^{+}((0,1)) \quad \text { and } \quad x \in(0,1) .
$$


Lemma 4 [19]. Let $\alpha, \beta \in(0,1]$ such that $\alpha+\beta>1$ and $\varphi \in \mathcal{B}^{+}((0,1))$. Then we have

(i) For $(x, t) \in(0,1) \times(0,1)$, we have:

$$
\frac{\alpha+\beta-1}{\beta \Gamma(\alpha+\beta)} K(x, t) \leq H(x, t) \leq \frac{1}{\Gamma(\alpha+\beta)} K(x, t),
$$

where $K(x, t):=x^{\alpha-1}(1-t)^{\alpha+\beta-2}(1-\max (x, t))$.

In particular,

$$
\begin{aligned}
& \frac{\alpha+\beta-1}{\beta \Gamma(\alpha+\beta)} x^{\alpha-1}(1-x)(1-t)^{\alpha+\beta-1} \leq H(x, t) \\
& \quad \leq \frac{1}{\Gamma(\alpha+\beta)} x^{\alpha-1}(1-t)^{\alpha+\beta-2} \min (1-t, 1-x) .
\end{aligned}
$$

(ii) For any $\varphi \in \mathcal{B}^{+}((0,1))$, the function $x \rightarrow V \varphi(x)=\int_{0}^{1} H(x, t) \varphi(t) \mathrm{d} t$ belongs to $C_{1-\alpha}([0,1])$ if and only if $\int_{0}^{1}(1-t)^{\alpha+\beta-1} \varphi(t) \mathrm{d} t<\infty$.

Next, we establish the following property of $H(x, t)$.

Proposition 1. We have, for $x, r, t \in(0,1)$,

$$
\frac{H(x, r) H(r, t)}{H(x, t)} \leq \frac{\beta}{(\alpha+\beta-1) \Gamma(\alpha+\beta)} r^{\alpha-1}(1-r)^{\alpha+\beta-1} .
$$

Proof. Using Lemma 4 (i), we have for each $x, r, t \in(0,1)$,

$$
\frac{H(x, r) H(r, t)}{H(x, t)} \leq \frac{\beta r^{\alpha-1}(1-r)^{\alpha+\beta-2}}{(\alpha+\beta-1) \Gamma(\alpha+\beta)} \frac{(1-\max (x, r))(1-\max (t, r))}{1-\max (x, t)} .
$$

We claim that

$$
\frac{(1-\max (x, r))(1-\max (t, r))}{1-\max (x, t)} \leq 1-r .
$$

Indeed, by symmetry, we may assume that $x \leq t$. Then we deduce that

$$
\begin{aligned}
\frac{(1-\max (x, r))(1-\max (t, r))}{1-t} & \leq 1-\max (x, r) \\
& \leq 1-r .
\end{aligned}
$$

Now, by using (2.5), we obtain the required result.

Next, we recall that $\omega(x):=a h_{1}(x)+b h_{2}(x)$, where

$$
h_{1}(x)=\frac{\Gamma(\beta)}{\Gamma(\alpha+\beta)} x^{\alpha-1}\left(1-x^{\beta}\right) \text { and } h_{2}(x)=x^{\alpha-1} \text {, for } x \in(0,1) .
$$

Proposition 2. Let $\alpha, \beta \in(0,1]$ such that $\alpha+\beta>1$ and let $q \in \mathcal{B}((0,1))$. Then we have

(i)

$$
\kappa_{q} \leq \frac{\beta}{(\alpha+\beta-1) \Gamma(\alpha+\beta)} \int_{0}^{1} r^{\alpha-1}(1-r)^{\alpha+\beta-1}|q(r)| \mathrm{d} r
$$

where $\kappa_{q}$ is given by (1.4). 
In particular, if $q \in \mathcal{K}_{\alpha, \beta}$, then $\kappa_{q}<\infty$.

(ii) For $x \in(0,1]$, we have

$$
\int_{0}^{1} H(x, t) h_{1}(t)|q(t)| \mathrm{d} t \leq \kappa_{q} h_{1}(x)
$$

and

$$
\int_{0}^{1} H(x, t) h_{2}(t)|q(t)| \mathrm{d} t \leq \kappa_{q} h_{2}(x) .
$$

In particular for $x \in(0,1]$,

$$
\int_{0}^{1} H(x, t) \omega(t)|q(t)| \mathrm{d} t \leq \kappa_{q} \omega(x) .
$$

Proof. Let $q$ be a function in $\mathcal{B}((0,1))$.

(i) The inequality (2.6) follows immediately from (1.4) and (2.4).

(ii) Since for each $x, t \in(0,1)$, we have $\lim _{r \rightarrow 0} \frac{H(t, r)}{H(x, r)}=\frac{H(t, 0)}{H(x, 0)}=\frac{h_{1}(t)}{h_{1}(x)}$, then we deduce by Fatou's lemma and (1.4) that

$$
\int_{0}^{1} H(x, t) \frac{h_{1}(t)}{h_{1}(x)}|q(t)| \mathrm{d} t \leq \liminf _{r \rightarrow 0} \int_{0}^{1} H(x, t) \frac{H(t, r)}{H(x, r)}|q(t)| \mathrm{d} t \leq \kappa_{q},
$$

which implies that for $x \in(0,1)$,

$$
\int_{0}^{1} H(x, t) h_{1}(t)|q(t)| \mathrm{d} t \leq \kappa_{q} h_{1}(x) .
$$

Similarly, we prove inequality $(2.8)$ by observing that

$$
\lim _{r \rightarrow 1} \frac{H(t, r)}{H(x, r)}=\frac{h_{2}(t)}{h_{2}(x)} .
$$

Inequality (2.9) follows from (2.7) to (2.8) and the fact that $\omega(x)=a h_{1}(x)+$ $b h_{2}(x)$.

Proposition 3 [19]. Let $\alpha, \beta \in(0,1]$ such that $\alpha+\beta>1$. Let $f \in C((0,1))$ such that the map $t \rightarrow(1-t)^{\alpha+\beta-1} f(t)$ is integrable and $|f(t)| \leq t^{-\delta} L(t)$ near 0 , with $\delta \leq 1$ and $L \in \mathcal{K}$ satisfying $\int_{0}^{\eta} t^{-\delta} L(t) \mathrm{d} t<\infty$. Then $V f$ is the unique solution in $C_{1-\alpha}([0,1])$ of the boundary value problem:

$$
\begin{cases}D^{\beta}\left(D^{\alpha}\right) u(x)=-f(x), & x \in(0,1), \\ \lim _{x \longrightarrow 0^{+}} x^{1-\beta} D^{\alpha} u(x)=0, & u(1)=0 .\end{cases}
$$

\section{Green's Function of the Operator $-D^{\beta}\left(D^{\alpha} u\right)+q(x) u$}

In this section, we will prove that the operator $-D^{\beta}\left(D^{\alpha} u\right)+q(x) u$ has a Green function for small nonnegative function $q$ in $\mathcal{K}_{\alpha, \beta}$. To this end, we need the following preliminary result. Let $\alpha, \beta \in(0,1]$ such that $\alpha+\beta>1$ and let $q \in \mathcal{B}^{+}((0,1))$. For $(x, t) \in(0,1] \times(0,1]$, put $H_{0}(x, t)=H(x, t)$ and

$$
H_{n}(x, t)=\int_{0}^{1} H(x, r) H_{n-1}(r, t) q(r) \mathrm{d} r, \quad n \geq 1 .
$$


Now, let $\mathcal{H}:(0,1] \times(0,1] \longrightarrow \mathbb{R}$ be defined by

$$
\mathcal{H}(x, t)=\sum_{n=0}^{\infty}(-1)^{n} H_{n}(x, t) .
$$

For $f \in B^{+}((0,1))$ and $x \in(0,1)$, we put

$$
V_{q} f(x):=\int_{0}^{1} \mathcal{H}(x, t) f(t) \mathrm{d} t .
$$

Then, we have the following.

Lemma 5. Let $\alpha, \beta \in(0,1]$ such that $\alpha+\beta>1$ and let $q$ be a nonnegative function in $\mathcal{K}_{\alpha, \beta}$ with $\kappa_{q}<1$. Then for $(x, t) \in(0,1] \times(0,1]$, we have

(i) For each $n \in \mathbb{N}$,

$$
H_{n}(x, t) \leq \kappa_{q}^{n} H(x, t) .
$$

In particular, $\mathcal{H}(x, t)$ is well defined on $(0,1] \times[0,1]$.

(ii) For each $n \in \mathbb{N}$, we have

$$
\begin{aligned}
& L_{n} x^{\alpha-1}(1-x)(1-t)^{\alpha+\beta-1} \leq H_{n}(x, t) \\
& \quad \leq R_{n} x^{\alpha-1}(1-t)^{\alpha+\beta-2} \min (1-t, 1-x),
\end{aligned}
$$

where

$$
L_{n}=\left(\frac{\alpha+\beta-1}{\beta \Gamma(\alpha+\beta)}\right)^{n+1}\left(\int_{0}^{1} r^{\alpha-1}(1-r)^{\alpha+\beta} q(r) \mathrm{d} r\right)^{n}
$$

and

$$
R_{n}=\frac{1}{(\Gamma(\alpha+\beta))^{n+1}}\left(\int_{0}^{1} r^{\alpha-1}(1-r)^{\alpha+\beta-1} q(r) \mathrm{d} r\right)^{n} .
$$

(iii) For $(x, t) \in(0,1] \times[0,1]$, we have

$$
\int_{0}^{1} \mathcal{H}(x, r) H(r, t) q(r) \mathrm{d} r=\int_{0}^{1} H(x, r) \mathcal{H}(r, t) q(r) \mathrm{d} r .
$$

Proof. The assertions (i) and (ii) are obtained by simple induction. Let us prove (iii). Let $n \geq 0$ and $x, t, r \in(0,1]$. By $(3.3)$, we have

$$
0 \leq H_{n}(x, t) H(r, t) q(r) \leq \kappa_{q}^{n} H(x, r) H(r, t) q(r) .
$$

Hence, the $\sum_{n \geq 0} \int_{0}^{1} H_{n}(x, r) H(r, t) q(r) \mathrm{d} r$ converges. So by the dominated convergence theorem, we deduce that

$$
\begin{aligned}
\int_{0}^{1} \mathcal{H}(x, r) H(r, t) q(r) \mathrm{d} r & =\sum_{n=0}^{\infty}(-1)^{n} \int_{0}^{1} H_{n}(x, r) H(r, t) q(r) \mathrm{d} r \\
& =\sum_{n=0}^{\infty}(-1)^{n} \int_{0}^{1} H(x, r) H_{n}(r, t) q(r) \mathrm{d} r \\
& =\int_{0}^{1} H(x, r) \mathcal{H}(r, t) q(r) \mathrm{d} r .
\end{aligned}
$$


Proposition 4. Let $\alpha, \beta \in(0,1]$ such that $\alpha+\beta>1$ and let $q$ be a nonnegative function in $\mathcal{K}_{\alpha, \beta}$ with $\kappa_{q}<1$. Then the function $(x, t) \rightarrow x^{1-\alpha} \mathcal{H}(x, t)$ is continuous on $[0,1] \times[0,1]$.

Proof. Firstly, we claim that for $n \in \mathbb{N}$, the function $(x, t) \rightarrow x^{1-\alpha} H_{n}(x, t)$ is continuous on $[0,1] \times[0,1]$. Indeed, from $(2.1)$ the function $(x, t) \rightarrow x^{1-\alpha} H_{0}$ $(x, t)$ is continuous on $[0,1] \times[0,1]$.

Assume that the function $(x, t) \rightarrow x^{1-\alpha} H_{n-1}(x, t)$ is continuous on $[0,1] \times[0,1]$.

Using Lemmas $5(\mathrm{i})$ and $4(\mathrm{i})$, we have for all $(x, t) \in[0,1] \times[0,1]$ and $r \in(0,1)$,

$$
\begin{aligned}
x^{1-\alpha} H(x, r) H_{n-1}(r, t) q(r) \leq & \kappa_{q}^{n-1} x^{1-\alpha} H(x, r) H(r, t) q(r) \\
\leq & \frac{1}{(\Gamma(\alpha+\beta))^{2}}(1-t)^{\alpha+\beta-2} \\
& \times \min (1-t, 1-x) r^{\alpha-1}(1-r)^{\alpha+\beta-1} q(r) \\
\leq & \frac{1}{(\Gamma(\alpha+\beta))^{2}} r^{\alpha-1}(1-r)^{\alpha+\beta-1} q(r) .
\end{aligned}
$$

Then since $q \in \mathcal{K}_{\alpha, \beta}$, we deduce by the dominated convergence theorem that the function $(x, t) \rightarrow x^{1-\alpha} H_{n}(x, t)$ is continuous on $[0,1] \times[0,1]$. This proves our claim.

Now, by using again Lemmas $5(\mathrm{i})$ and $4(\mathrm{i})$, we have for each $x, t \in[0,1]$,

$$
x^{1-\alpha} H_{n}(x, t) \leq \kappa_{q}^{n} x^{1-\alpha} H(x, t) \leq \frac{1}{\Gamma(\alpha+\beta)} \kappa_{q}^{n} .
$$

This implies that the series $\sum_{n \geq 0}(-1)^{n} H_{n}(x, t)$ uniformly converges on $[0,1] \times$ $[0,1]$ and therefore the function $(x, t) \rightarrow x^{1-\alpha} \mathcal{H}(x, t)$ is continuous on $[0,1] \times$ $[0,1]$. The proof is completed.

Lemma 6. Let $\alpha, \beta \in(0,1]$ such that $\alpha+\beta>1$ and let $q$ be a nonnegative function in $\mathcal{K}_{\alpha, \beta}$ with $\kappa_{q} \leq \frac{1}{2}$. Then for $(x, t) \in(0,1] \times[0,1]$, we have

$$
\left(1-\kappa_{q}\right) H(x, t) \leq \mathcal{H}(x, t) \leq H(x, t) .
$$

Proof. Since $\kappa_{q} \leq \frac{1}{2}$, we deduce from Lemma 5(i) that

$$
|\mathcal{H}(x, t)| \leq \sum_{n=0}^{\infty} \kappa_{q}^{n} H(x, t)=\frac{1}{1-\kappa_{q}} H(x, t) .
$$

On the other hand, from the expression of $\mathcal{H}$ we have

$$
\mathcal{H}(x, t)=H(x, t)-\sum_{n=0}^{\infty}(-1)^{n} H_{n+1}(x, t) .
$$

Since the series $\sum_{n \geq 0} \int_{0}^{1} H(x, r) H_{n}(r, t) q(r) \mathrm{d} r$ is convergent, we deduce by (3.8) and (3.2) that 


$$
\begin{aligned}
\mathcal{H}(x, t) & =H(x, t)-\sum_{n=0}^{\infty}(-1)^{n} \int_{0}^{1} H(x, r) H_{n}(r, t) q(r) \mathrm{d} r \\
& =H(x, t)-\int_{0}^{1} H(x, r)\left(\sum_{n=0}^{\infty}(-1)^{n} H_{n}(r, t)\right) q(r) \mathrm{d} r .
\end{aligned}
$$

That is,

$$
\mathcal{H}(x, t)=H(x, t)-V(q \mathcal{H}(., t))(x) .
$$

On the other hand, since

$$
\begin{aligned}
V(q \mathcal{H}(., t))(x) & \leq \frac{1}{1-\kappa_{q}} V(q H(., t))(x) \\
& =\frac{1}{1-\kappa_{q}} H_{1}(x, t) \\
& \leq \frac{\kappa_{q}}{1-\kappa_{q}} H(x, t),
\end{aligned}
$$

we deduce that

$$
\mathcal{H}(x, t) \geq H(x, t)-\frac{\kappa_{q}}{1-\kappa_{q}} H(x, t)=\frac{1-2 \kappa_{q}}{1-\kappa_{q}} H(x, t) \geq 0 .
$$

So it follows that $0 \leq \mathcal{H}(x, t) \leq H(x, t)$ and by $(3.9)$

$$
\mathcal{H}(x, t) \geq H(x, t)-V(q H(., t))(x) \geq\left(1-\kappa_{q}\right) H(x, t) .
$$

Corollary 1. Let $\alpha, \beta \in(0,1]$ such that $\alpha+\beta>1$ and let $q$ be a nonnegative function in $\mathcal{K}_{\alpha, \beta}$ with $\kappa_{q} \leq \frac{1}{2}$. Let $f \in \mathcal{B}^{+}((0,1))$, then

$$
V_{q} f(x) \in C_{1-\alpha}([0,1]) \text { if and only if } \int_{0}^{1}(1-t)^{\alpha+\beta-1} f(t) \mathrm{d} t<\infty \text {. }
$$

Next, we will prove that the kernel $V_{q}$ satisfies the following resolvent equation.

Lemma 7. Let $\alpha, \beta \in(0,1]$ such that $\alpha+\beta>1$ and let $q$ be a nonnegative function in $\mathcal{K}_{\alpha, \beta}$ with $\kappa_{q} \leq \frac{1}{2}$. Let $f \in \mathcal{B}^{+}((0,1))$, then $V_{q} f$ satisfies the following resolvent equation:

$$
V f=V_{q} f+V_{q}(q V f)=V_{q} f+V\left(q V_{q} f\right) .
$$

In particular, if $V(q f)<\infty$, we have

$$
\left(I-V_{q}(q .)\right)(I+V(q .)) f=(I+V(q .))\left(I-V_{q}(q .)\right) f=f .
$$

Proof. Let $(x, t) \in(0,1] \times(0,1]$, then by $(3.9)$, we have

$$
H(x, t)=\mathcal{H}(x, t)+V(q \mathcal{H}(., t))(x),
$$

which implies by the Fubini-Tonelli theorem that for $f \in \mathcal{B}^{+}((0,1))$,

$$
\begin{aligned}
V f(x) & =\int_{0}^{1}(\mathcal{H}(x, t)+V(q \mathcal{H}(., t))(x)) f(t) \mathrm{d} t \\
& =V_{q} f(x)+V\left(q V_{q} f\right)(x) .
\end{aligned}
$$


On the other hand, by Lemma 5(iii) and again the Fubini-Tonelli theorem, we have

$$
\int_{0}^{1} \int_{0}^{1} \mathcal{H}(x, r) H(r, t) q(r) f(t) \mathrm{d} r \mathrm{~d} t=\int_{0}^{1} \int_{0}^{1} H(x, r) \mathcal{H}(r, t) q(r) f(t) \mathrm{d} r \mathrm{~d} t .
$$

That is,

So, we obtain

$$
V_{q}(q V f)(x)=V\left(q V_{q} f\right)(x)
$$

$$
V f=V_{q} f+V_{q}(q V f)=V_{q} f+V\left(q V_{q} f\right) .
$$

Proposition 5. Let $\alpha, \beta \in(0,1]$ such that $\alpha+\beta>1$ and let $q$ be a nonnegative function in $\mathcal{K}_{\alpha, \beta} \cap C((0,1))$ satisfying (i) and (ii) in $\left(H_{2}\right)$. Let $f \in C^{+}((0,1))$ such that the map $t \rightarrow(1-t)^{\alpha+\beta-1} f(t)$ is integrable and $f(t) \leq t^{-\delta} \tilde{L}(t)$ near 0 , with $\delta \leq 1$ and $\tilde{L} \in \mathcal{K}$ satisfying $\int_{0}^{\eta} t^{-\delta} \tilde{L}(t) \mathrm{d} t<\infty$. Then, $V_{q} f \in$ $C_{1-\alpha}([0,1])$ and it is the unique nonnegative solution of the perturbed problem

$$
\left\{\begin{array}{l}
D^{\beta}\left(D^{\alpha}\right) u(x)-q(x) u(x)=-f(x), \quad x \in(0,1), \\
\lim _{x \longrightarrow 0^{+}} x^{1-\beta} D^{\alpha} u(x)=u(1)=0,
\end{array}\right.
$$

satisfying

$$
\left(1-\kappa_{q}\right) V f \leq V_{q} f \leq V f .
$$

Proof. Since by Corollary 1 the function $t \rightarrow V_{q} f(t)$ is in $C_{1-\alpha}([0,1])$, it follows that the function $t \rightarrow q(t) V_{q} f(t)$ is continuous on $(0,1)$. that

Using (3.6) and Lemma 4(i), there exists a nonnegative constant $c$ such

$$
\begin{aligned}
V_{q} f(x) & \leq V f(x) \\
& \leq \frac{1}{\Gamma(\alpha+\beta)} x^{\alpha-1} \int_{0}^{1}(1-t)^{\alpha+\beta-2} \min (1-t, 1-x) f(t) \mathrm{d} t \\
& \leq \frac{1}{\Gamma(\alpha+\beta)} x^{\alpha-1} \int_{0}^{1}(1-t)^{\alpha+\beta-1} f(t) \mathrm{d} t \\
& \leq c x^{\alpha-1} .
\end{aligned}
$$

Therefore,

$$
\int_{0}^{1}(1-t)^{\alpha+\beta-1} q(t) V_{q} f(t) \mathrm{d} t \leq c \int_{0}^{1} t^{\alpha-1}(1-t)^{\alpha+\beta-1} q(t) \mathrm{d} t<\infty
$$

and $q(t) V_{q} f(t) \leq c t^{\alpha-1} q(t) \leq c t^{-\mu} L(t)$ for $t$ near 0 , where $\mu \leq 1$ and $L \in \mathcal{K}$ satisfies $\int_{0}^{\eta} t^{-\mu} L(t) \mathrm{d} t<\infty$. Hence by using Proposition 3 , we conclude that the function $u=V_{q} f=V f-V\left(q V_{q} f\right)$ satisfies

$$
\left\{\begin{array}{l}
D^{\beta}\left(D^{\alpha}\right) u(x)=-f(x)+q(x) u(x), \quad x \in(0,1), \\
\lim _{x \longrightarrow 0^{+}} x^{1-\beta} D^{\alpha} u(x)=u(1)=0 .
\end{array}\right.
$$

It remains to prove the uniqueness. Assume that there exists another nonnegative solution $v$ in $C_{1-\alpha}([0,1])$ of problem (3.12) satisfying (3.13). 
We remark that the function $t \rightarrow q(t) v(t)$ is continuous on $(0,1)$ and by (3.13) and (2.3) we have

$$
\begin{aligned}
\int_{0}^{1}(1-t)^{\alpha+\beta-1} q(t) v(t) \mathrm{d} t & \leq \int_{0}^{1}(1-t)^{\alpha+\beta-1} q(t) V f(t) \mathrm{d} t \\
& \leq c \int_{0}^{1} t^{\alpha-1}(1-t)^{\alpha+\beta-1} q(t) \mathrm{d} t<\infty
\end{aligned}
$$

Moreover, we have

$$
q(t) v(t) \leq q(t) V f(t) \leq c t^{\alpha-1} q(t) \leq c t^{-\mu} L(t) \quad \text { for } t \text { near } 0,
$$

where $\mu \leq 1$ and $L \in \mathcal{K}$ satisfies $\int_{0}^{\eta} t^{-\mu} L(t) \mathrm{d} t<\infty$.

It follows by Proposition 3, the function $\tilde{v}:=v+V(q v)$ satisfies

$$
\left\{\begin{array}{l}
D^{\beta}\left(D^{\alpha}\right) \tilde{v}(x)=-f(x), \quad x \in(0,1), \\
\lim _{x \longrightarrow 0^{+}} x^{1-\beta} D^{\alpha} \tilde{v}(x)=\tilde{v}(1)=0 .
\end{array}\right.
$$

From the uniqueness in Proposition 3, we deduce that $\tilde{v}:=v+V(q v)=V f$.

Hence,

$$
(I+V(q \cdot))(v-u)=0 .
$$

Now, since for $x \in(0,1]$, there exists a nonnegative constant $c$ such that $V_{q} f(x) \leq V f(x) \leq c h_{2}(x)$, where $h_{2}$ given by (1.6), it follows by (2.8) that

$$
V(q|v-u|) \leq 2 c V\left(q h_{2}\right) \leq 2 c \kappa_{q} h_{2}<\infty .
$$

So by (3.11), we deduce that $u=v$.

\section{Proof of Theorem 1}

Consider $a \geq 0$ and $b \geq 0$ such that $a+b>0$. Let $\alpha, \beta \in(0,1]$ such that $\alpha+\beta>1$, and $q \in \mathcal{K}_{\alpha, \beta} \cap C((0,1))$ be such that $\left(H_{2}\right)$ is satisfied.

Let

$$
\Lambda:=\left\{u \in \mathcal{B}^{+}((0,1)):\left(1-\kappa_{q}\right) \omega \leq u \leq \omega\right\},
$$

where $\omega(x)=a h_{1}(x)+b h_{2}(x), h_{1}$ and $h_{2}$ are defined by (1.6).

Define the operator $T$ on $\Lambda$ by

$$
T u=\omega-V_{q}(q \omega)+V_{q}((q-g(u)) u) .
$$

By (3.6) and (2.9), we have

$$
V_{q}(q \omega) \leq V(q \omega) \leq \kappa_{q} \omega \leq \omega .
$$

Using $\left(H_{2}\right)$, we get

$$
0 \leq g(u) \leq q \quad \text { for all } u \in \Lambda .
$$

Next, we prove that $\Lambda$ is invariant under $T$. Indeed, using (4.1) and (4.2), we have for $u \in \Lambda$,

$$
T u \leq \omega-V_{q}(q \omega)+V_{q}(q u) \leq \omega
$$

and

$$
T u \geq \omega-V_{q}(q \omega) \geq\left(1-\kappa_{q}\right) \omega .
$$


Next, we will prove that the operator $T$ is nondecreasing on $\Lambda$. Indeed, let $u, v \in \Lambda$ be such that $u \leq v$. By $\left(H_{2}\right)$, the function $t \rightarrow t(q(x)-g(t \omega))$ is nondecreasing on $[0,1]$, for $x \in(0,1)$.

Then, we obtain

$$
T v-T u=V_{q}([v(q-g(v))-u(q-g(u))]) \geq 0 .
$$

Now, define the sequence $\left(u_{n}\right)$ by $u_{0}=\left(1-\kappa_{q}\right) \omega$ and $u_{n+1}=T u_{n}$ for $n \in \mathbb{N}$.

Since $T \Lambda \subseteq \Lambda$, we have $u_{1}=T u_{0} \geq u_{0}$ and, by the monotonicity of $T$, we deduce that

$$
\left(1-\kappa_{q}\right) \omega=u_{0} \leq u_{1} \leq \cdots \leq u_{n} \leq u_{n+1} \leq \omega .
$$

Hence by $\left(H_{1}\right)-\left(H_{2}\right)$ and the dominated convergence theorem, we deduce that the sequence $\left(u_{n}\right)$ converges to a function $u \in \Lambda$ satisfying

$$
u=\left(I-V_{q}(q .)\right) \omega+V_{q}((q-g(u)) u),
$$

that is,

$$
\left(I-V_{q}(q .)\right) u=\left(I-V_{q}(q .)\right) \omega-V_{q}(u g(u)) .
$$

By (2.9), we have $V(q u) \leq V(q \omega) \leq \omega<\infty$; then applying the operator $(I+V(q)$.$) on both sides of the above equality and using (3.10) and (3.11),$ we deduce that $u$ satisfies

$$
u=\omega-V(u g(u)) .
$$

It remains to prove that $u$ is a solution of problem (1.3). Using (4.2) and (1.6), we have

$$
0 \leq u(t) g(u(t)) \leq q(t) \omega(t) \leq \max \left(\frac{\Gamma(\beta)}{\Gamma(\alpha+\beta)} a, b\right) t^{\alpha-1} q(t) .
$$

Therefore,

$$
\begin{aligned}
\int_{0}^{1}(1-t)^{\alpha+\beta-1} u(t) g(u(t)) \mathrm{d} t \leq & \max \left(\frac{\Gamma(\beta)}{\Gamma(\alpha+\beta)} a, b\right) \\
& \int_{0}^{1} t^{\alpha-1}(1-t)^{\alpha+\beta-1} q(t) \mathrm{d} t<\infty .
\end{aligned}
$$

So, by Lemma 4 (ii) the function $x \rightarrow V(u g(u))(x)$ is in $C_{1-\alpha}([0,1])$. This implies by $(4.3), u \in C_{1-\alpha}([0,1])$. Now, we remark that the function $t \rightarrow$ $(1-t)^{\alpha+\beta-1} u(t) g(u(t))$ is continuous and integrable on $(0,1)$. Moreover, by (4.2) and $\left(H_{2}\right)$, we have for $t$ near 0

$$
0 \leq u(t) g(u(t)) \leq \max \left(\frac{\Gamma(\beta)}{\Gamma(\alpha+\beta)} a, b\right) t^{-\mu} L(t),
$$

where $\mu \leq 1$ and $L \in \mathcal{K}$ satisfies $\int_{0}^{\eta} t^{-\mu} L(t) \mathrm{d} t<\infty$.

Then, we deduce by Proposition 3 that $u$ is a solution of (1.3).

Finally, suppose that hypotheses $\left(H_{3}\right)$ is satisfied and let us show that problem (1.3) has a unique solution satisfying (1.7). Assume that $v$ is another nonnegative solution in $C_{1-\alpha}([0,1])$ to problem $(1.3)$ satisfying the inequality $(1.7)$. 
Since $v \leq \omega$, we deduce by (4.2) that

$$
0 \leq v(t) g(v(t)) \leq q(t) \omega(t) \leq \max \left(\frac{\Gamma(\beta)}{\Gamma(\alpha+\beta)} a, b\right) t^{\alpha-1} q(t) .
$$

This implies that, the function $t \rightarrow(1-t)^{\alpha+\beta-1} v(t) g(v(t)) \in L^{1}((0,1)) \cap$ $C((0,1))$ and for $t$ near 0 , we have

$$
0 \leq v(t) g(v(t)) \leq \max \left(\frac{\Gamma(\beta)}{\Gamma(\alpha+\beta)} a, b\right) t^{-\mu} L(t),
$$

where $\mu \leq 1$ and $L \in \mathcal{K}$ satisfies $\int_{0}^{\eta} t^{-\mu} L(t) \mathrm{d} t<\infty$. Let $\tilde{v}:=v+V(v g(v))$.

By Proposition 3, we have

$$
\left\{\begin{array}{l}
D^{\beta}\left(D^{\alpha}\right) \tilde{v}(x)=0, x \in(0,1), \\
\lim _{x \longrightarrow 0^{+}} x^{1-\beta} D^{\alpha} \tilde{v}(x)=-a, \quad \tilde{v}(1)=b .
\end{array}\right.
$$

Hence,

$$
v=\omega-V(v g(v))
$$

Now, let be $h:(0,1) \rightarrow \mathbb{R}$ defined by

$$
h(t):= \begin{cases}\frac{v(t) g(v(t))-u(t) g(u(t))}{v(t)-u(t)}, & \text { if } v(t) \neq u(t), \\ 0, & \text { if } v(t)=u(t) .\end{cases}
$$

By $\left(H_{3}\right), h \in \mathcal{B}^{+}((0,1))$ and from $(4.3)$ and $(4.5)$, we deduce that

$$
(I+V(h .))(v-u)=0 .
$$

From $\left(H_{2}\right)$, we have $h \leq q$. So by using (2.9), we deduce that

$$
\begin{aligned}
V(h|v-u|) & \leq V(v g(v))+V(u g(u)) \\
& \leq 2 V(q \omega) \\
& \leq 2 \kappa_{q} \omega<\infty .
\end{aligned}
$$

Hence by (3.11), we conclude that $u=v$. This completes the proof.

To illustrate our result proved in Theorem 1, we give the following example.

Example 1. Let $\alpha, \beta \in(0,1]$ such that $\alpha+\beta>1$, and let $\sigma \geq 1$ and $a \geq 0$, $b \geq 0$ such that $a+b>0$. Then for nonnegative small $\lambda$, the following problem

$$
\left\{\begin{array}{l}
D^{\beta}\left(D^{\alpha} u\right)(x)=\lambda u^{\sigma}(x), 0<x<1, \\
\lim _{x \rightarrow 0^{+}} x^{1-\beta} D^{\alpha} u(x)=-a, u(1)=b,
\end{array}\right.
$$

has a unique positive solution $u$ in $C_{1-\alpha}([0,1])$ satisfying

$$
c \omega(x) \leq u(x) \leq \omega(x) \quad \text { for } \quad x \in(0,1),
$$

where $0<c<1$.

\section{Acknowledgements}

We thank the referee for his/her careful reading of the paper and helpful comments and remarks. 
Open Access. This article is distributed under the terms of the Creative Commons Attribution 4.0 International License (http://creativecommons.org/licenses/ by/4.0/), which permits unrestricted use, distribution, and reproduction in any medium, provided you give appropriate credit to the original author(s) and the source, provide a link to the Creative Commons license, and indicate if changes were made.

\section{References}

[1] Bachar, I., Mâagli, H., Rădulescu, V.D.: Fractional Navier boundary value problems. Bound. Value Probl. 20, 79 (2016)

[2] Campos, L.M.C.M.: On the solution of some simple fractional differential equations. Int. J. Math. Sci. 13, 481-496 (1990)

[3] Chaieb, M., Dhifli, A., Zribi, M.: Positive solutions for systems of competitive fractional differential equations. Electron. J. Differ. Equ. 2016(133), 1-13 (2016)

[4] Delbosco, D., Rodino, L.: Existence and uniqueness for a nonlinear fractional differential equation. J. Math. Anal. Appl. 204, 609-625 (1996)

[5] Diethelm, K., Freed, A.D.: On the solution of nonlinear fractional order differential equations used in the modeling of viscoplasticity. In: Keil, F., Mackens, W., Voss, H., Werther, J. (eds.) Scientific Computing in Chemical Engineering II-Computational Fluid Dynamics, Reaction Engineering and Molecular Properties, pp. 217-224. Springer, Heidelberg (1999)

[6] Furati, K.M., Kassim, M.D., Tatar, N.-E.: Existence and uniqueness for a problem involving Hilfer fractional derivative. Comput. Math. Appl. 64, 1616-1626 (2012)

[7] Furati, K.M., Kassim, M.D., Tatar, N.-E.: Non-existence of global solutions for a differential equation involving Hilfer fractional derivative. Electron. J. Differ. Equ. 2013(235), 1-10 (2013)

[8] Hilfer, R.: Applications of Fractional Calculus in Physics. World Scientific, Singapore (2000)

[9] Kilbas, A.A., Srivastava, H.M., Trujillo, J.J.: Theory and Applications of Fractional Differential Equations. Elsevier, Amsterdam (2006)

[10] Kilbas, A.A., Trujillo, J.J.: Differential equations of fractional order methods, results and problems, I. Appl. Anal. 78, 153-197 (2001)

[11] Kilbas, A.A., Trujillo, J.J.: Differential equations of fractional order methods, results and problems, II. Appl. Anal. 81, 135-193 (2002)

[12] Koeller, R.C.: Applications of fractional calculus to the theory of viscoelasticity. J. Appl. Mech. 51(5), 299-307 (1984)

[13] Kou, C., Zhou, H., Yan, Y.: Existence of solutions of initial value problems for nonlinear fractional differential equations on the half-axis. Nonlinear Anal. 74, 5975-5986 (2011)

[14] Kristaly, A., Rădulescu, V.D., Varga, C.: Variational Principles in Mathematical Physics, Geometry, and Economics: Qualitative Analysis of Nonlinear Equations and Unilateral Problems, vol. 136. Cambridge University Press, Cambridge (2010) 
[15] Kumar, P., Agarwal, O.P.: An approximate method for numerical solution of fractional differential equations. Signal Process. 86, 2602-2610 (2006)

[16] Lakshmikantham, V., Vatsala, A.S.: General uniqueness and monotone iterative technique for fractional differential equations. Appl. Math. Lett. 21, 828834 (2008)

[17] Ling, Y., Ding, S.: A class of analytic functions defined by fractional derivation. J. Math. Anal. Appl. 186, 504-513 (1994)

[18] Mâagli, H., Chaieb, M., Dhifli, A., Zermani, S.: Existence and boundary behavior of positive solutions for a semilinear fractional differential equation. Mediterr. J. Math. 12, 1265-1285 (2015)

[19] Mâagli, H., Dhifli, A.: Existence and asymptotic behavior of positive solutions for semilinear fractional Navier boundary-value problems. Electron. J. Differ. Equ. 2017(141), 1-13 (2017)

[20] Maric, V.: Lecture Notes in Maths. Regular variation and differential equations, vol. 1726. Springer, Berlin (2000)

[21] Miller, K.S., Ross, B.: An Introduction to the Fractional Calculus and Fractional Differential Equations. Wiley, New York (1993)

[22] Pitcher, E., Sewell, W.E.: Existence theorems for solutions of differential equations of non-integral order. Bull. Am. Math. Soc. 44(2), 100-107 (1938)

[23] Podlubny, I.: Geometric and physical interpretation of fractional integration and fractional differentiation. Fract. Calc. Appl. Anal. 5, 367-386 (2002)

[24] Pucci, P., Xiang, M., Zhang, B.: Existence and multiplicity of entire solutions for fractional $p$-Kirchhoff equations. Adv. Nonlinear Anal. 5, 27-55 (2016)

[25] Rădulescu, V.D.: Qualitative Analysis of Nonlinear Elliptic Partial Differential Equations. Hindawi Publishing Corporation, Cairo (2008)

[26] Samko, S., Kilbas, A., Marichev, O.: Fractional integrals and Derivative. Theory and Applications. Gordon and Breach, Yverdon (1993)

[27] Seneta, R.: Lectures Notes in Mathematics. Regular varying functions, vol. 508. Springer, Berlin (1976)

[28] Tarasov, V.: Fractional Dynamics: Applications of Fractional Calculus to Dynamics of Particles. Fields and Media. Springer, New York (2011)

[29] Zhang, S.: The existence of a positive solution for a nonlinear fractional differential equation. J. Math. Anal. Appl. 252, 804-812 (2000)

[30] Zhang, S.: Monotone iterative method for initial value problem involving Riemann-Liouville fractional derivatives. Nonlinear Anal. 71, 2087-2093 (2009)

\author{
Habib Mâagli \\ Department of Mathematics, College of Sciences and Arts \\ King Abdulaziz University \\ Rabigh Campus \\ P.O. Box 344 \\ Rabigh 21911 \\ Saudi Arabia \\ e-mail: habib.maagli@fst.rnu.tn
}


Abdelwaheb Dhifli

Département de Mathématiques

Faculté des Sciences de Tunis

Campus Universitaire

2092 Tunis

Tunisia

e-mail: dhifli_waheb@yahoo.fr

Abdulah Khamis Alzahrani

Department of Mathematics, Faculty of Sciences

King Abdulaziz University

P.O. Box 80203

Jeddah 21589

Saudi Arabia

e-mail: akalzahrani@kau.edu.sa

Received: September 26, 2017.

Revised: February 28, 2018.

Accepted: March 15, 2018. 